\title{
Multi-objective optimization under uncertainty for sheet metal forming.
}

\author{
Pascal Lafon ${ }^{1, a}$, Pierre Antoine Adragna ${ }^{1}$, and Von Dim Nguyen ${ }^{2}$ \\ ${ }^{1}$ Université de technoglogie de Troyes ICD-LASMIS UMR CNRS 6281 \\ ${ }^{2}$ Université de technologie de l'information et de la communication de Thai Nguyen
}

\begin{abstract}
Aleatory uncertainties in material properties, blank thickness and friction condition are inherent and irreducible variabilities in sheet metal forming. Optimal design configurations, which are obtained by conventional design optimization methods, are not always able to meet the desired targets due to the effect of uncertainties. This paper proposes a multi-objective robust design optimization that aims to tackle this problem. Results obtained on a U shape draw bending benchmark show that spring-back effect can be controlled by optimizing process parameters.
\end{abstract}

\section{Introduction}

In Sheet metal forming processes, intrinsic variabilities in material properties, geometry of blank and process parameters are unavoidable. These variabilities will be considered here as aleatory uncertainties. As a consequence, these uncertainties may lead to instabilities in performance of manufactured parts such as shape variation due to spring-back, thinning, tearing and wrinkling. Here we are interested in instabilities produced by uncertainties on spring-back effects. We will consider the problem of tolerance specification in detailed design phase of stamped part. In this detailed design phase, to ensure the desired behaviour and functional requirements of a part in spite of uncertainties, the part features are assigned to a tolerance zone that must contain the part features. Therefore, tolerance design is a key element in industry for improving product quality and decreasing the manufacturing cost. Since the sheet metal part's performance variation induced by uncertainties of the inputs is quite large due to springback, geometric tolerance analysis for the sheet metal part is a problem to consider.

The goal of this paper is to propose a robust design optimization of forming processes. In this study we will take into account uncertainties of process parameter, material and geometry uncertainties of the blank. First we will introduce the two main approaches of dealing with uncertainties in an a optimization framework. Next, the general formulation of a robust optimization problem will be presented.

\section{Uncertainties, robust or reliable optimization of processes}

Dealing with uncertainties can be achieved by considering "Reliability Based Design Optimization (RBDO)" or "Ro- bust Design Optimization (RDO)". Reliability analysis and optimization are two essential components of RBDO: (1) Reliability Analysis focuses on analyzing the probabilistic constraints to ensure the reliability levels are satisfied; (2) Optimization is seeking for the optimal performance subject to the probabilistic constraints. The RBDO approach handles noise variables in a probabilistic way. The RBDO was mainly used in optimization under uncertainty of sheet metal forming processes which the failure probability of wrinkling or excessive thinning was considered [1-4].Robust Design Optimization (RDO) aims at minimizing the variability of the part performance, while meeting the requirements of optimum performance and constraint conditions. Similar to the RBDO approach, uncertainties are handled in a probabilistic way. There is still a disagreement relating to the similarity between the RBDO and the RDO. According to Park et al. [5], the RBDO is similar to the RDO. Both approaches aim at incorporating uncertainty into the optimization study. Whereas Zang et al. [6] discussed that there is a conceptual difference between RDO and RBDO. The RDO rather aims at reducing the variability of performance caused by fluctuations in parameters than to avoid a catastrophe in an extreme event. In the case of RBDO, it can make a design that displays large variations as long as there are safety margins failure in the design, i.e. the variability is not minimized. Furthermore, the two approaches differ in some aspects [7]. The choice for using the RDO approach or the RBDO approach depends on the objective of the optimization study. In the RDO, insensitiveness of the objective function is emphasized. In the RBDO, reliability of constraints is important [5]. Thus, both approaches will obviously lead to different optimization outcomes.

\footnotetext{
a corresponding author :pascal.lafon@utt.fr
} 


\section{A general framework for robust optimization}

We consider the robust optimization state as the following multi-objective optimization problem:

$$
\left\{\begin{array}{l}
\min _{X} E[F(X, P)] \\
\text { and } \\
\min _{X} \sigma[F(X, P)] \\
\text { Under the constraints } \\
\mathcal{P}[C(X, P) \leq 0] \geq \beta
\end{array}\right.
$$

Where $X$ and $P$ are vectors of random variables, which are modelled as normal probability distribution functions. Here $X$ represents the design variables, which are controllable by the optimization process, and $P$ represents the noise parameters which are not controllable. An expected value $\left(E\left[x_{i}\right], E\left[p_{i}\right]\right)$ and standard deviation $\left.\sigma\left[x_{i}\right]\right)$, $\left.\left.\sigma\left[p_{i}\right]\right)\right)$ are associated to each component $x_{i}$ and $p_{j}$ of these vector of variable. Here the criteria to be optimized $F(X, P)$ and the constraints $C(X, P)$ are written as a vector of function. In this formulation, $\mathcal{P}[C(X, P) \leq 0]$ is the probability to respect the event "all constraints $C(X, P)$ are respected, i.e less than 0 . In this kind of problem the evaluation of expected value $E[F(X, P)]$ and standard deviation $\sigma[F(X, P)]$ is known as "uncertainty propagation". This can be achieved by sampling methods (e.g. Monte-Carlo), approximation method like First or Second Order Method (FORM/SORM) or polynomial chaos. Practically, as soon as evaluation of function $F$ and $C$ requires finite elements calculation, surrogate model or meta model became essential. Thus, to obtain the solution for this kind of optimization problem, i.e the Pareto Front, we must go through the following steps:

- Setting up a "Design Of Experiment" on the domain of design variables $X$ and noise parameters $P$, considering here that we deal with deterministic variables and parameters.

- Building a suitable meta-model for each single function of the vector of functions $F(X, P)$ and $C(X, P)$.

- Embedding uncertainty propagation into an optimization framework : calculation of expected value and standard deviation using meta-model within an stochastic optimization algorithm like NGSAII.

This procedure is now implemented in some commercial software as for example ModeFrontier ${ }^{\mathrm{TM}}$ or Isight ${ }^{\mathrm{TM}}$. In this paper this procedure will be used on a NUMISHEET 2011 benchmark, and we will focus on spring-back effects for this draw bending process.

\section{Case study : U shaped draw bending}

The case study in this paper is a benchmark problem of NUMISHEET 2011 which the springback behavior of advanced high strength steels of DP780 steel in U-shaped draw bending is investigated [8]. The DP780 steel sheet with $1.4 \mathrm{~mm}$ thick, $360 \mathrm{~mm}$ long and $30 \mathrm{~mm}$ wide is considered. Experiments show that part profiles remain symmetric through the manufacturing process (see Fig. 1).

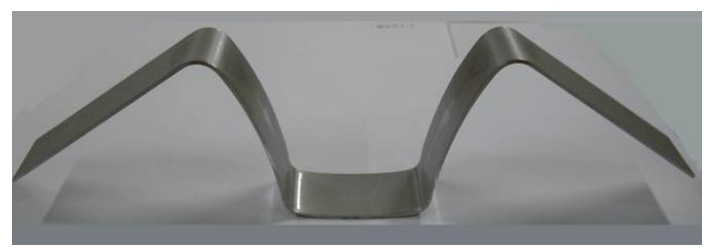

Figure 1. Effects of spring-back on U-shape drawing

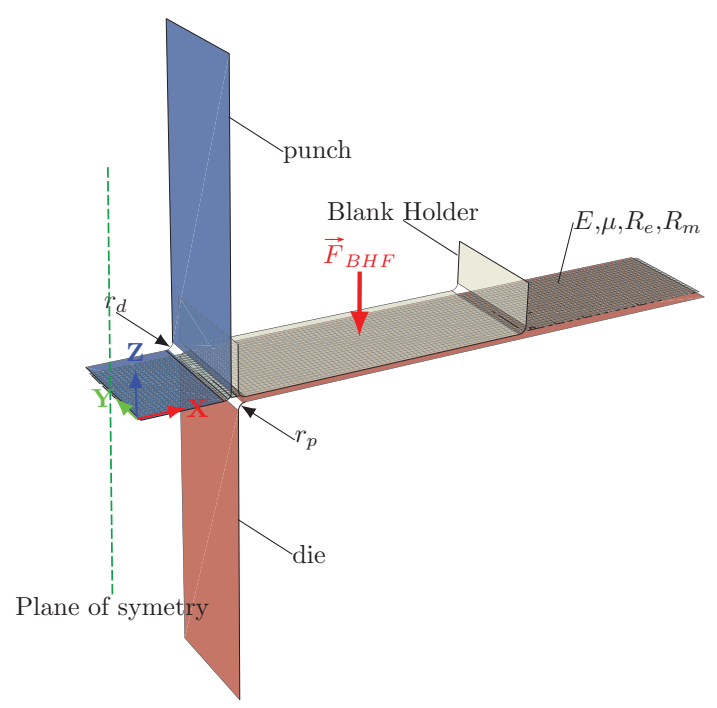

Figure 2. Numerical model of U-shape draw bending process

\subsection{Numerical model of the draw bending process}

The main tooling set-up and modelling for the draw bending process is shown in Fig. 2. In this study, modelling and numerical simulation of draw bending process are carried out by Finite Element Method using ABAQUS 6.12-2. The blank is modelled by 2709 S4R-shell elements with 7 integration points through thickness. The blank's strainhardening behaviour is described by Swift law. Yield behaviour of the material is modelled by Hill' 48 function. The nominal material properties of the blank are given as follows: Young's modulus $E=198.8 \mathrm{GPa}$, Yield strength $R_{e}=550 \mathrm{MPa}$, Tensile strength $R_{m}=840 \mathrm{MPa}$, Uniform elongation $\varepsilon_{u}=13.1 \%$, "R-value" $=0.781$, Poisson's ratio $v=0.3$. All tools are assumed to be rigid bodies. Boundary conditions applied on the tools are demonstrated in Fig.2. Contact interaction is modelled by penalty contact enforcement method in which type of contact of surfaceto-surface is applied between blank surface and the tool's surfaces.

The process is executed through two different steps. The first step is to simulate forming operation via dynamic explicit. The punch moves downward a travel of $71.8 \mathrm{~mm}$. Simulation of spring-back behaviour is performed in the second step through static implicit procedure. The final shape of the part is obtained after releasing the tools. An example of effects of spring-back is represent on Fig. 3. The U-shape after spring-back is defined by three parameters : the angles of $\theta_{1}, \theta_{2}$ and the side wall curl $\rho$ (see Fig. 4. 


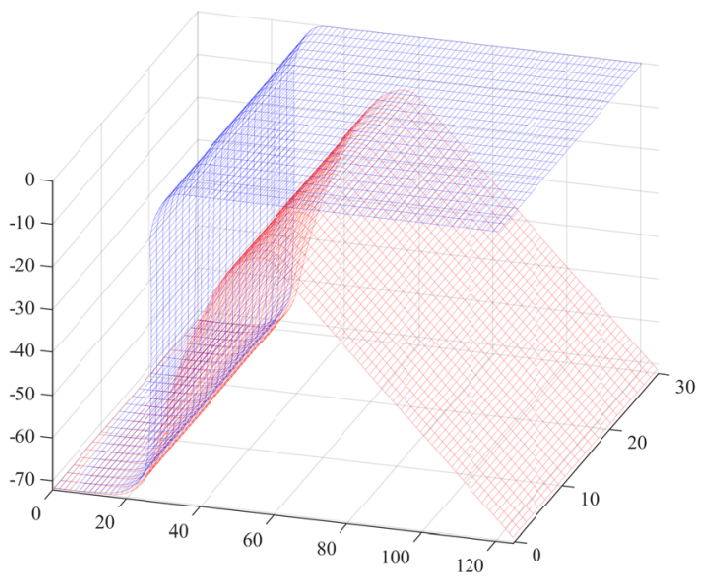

Figure 3. Example of the U-shape after spring-back

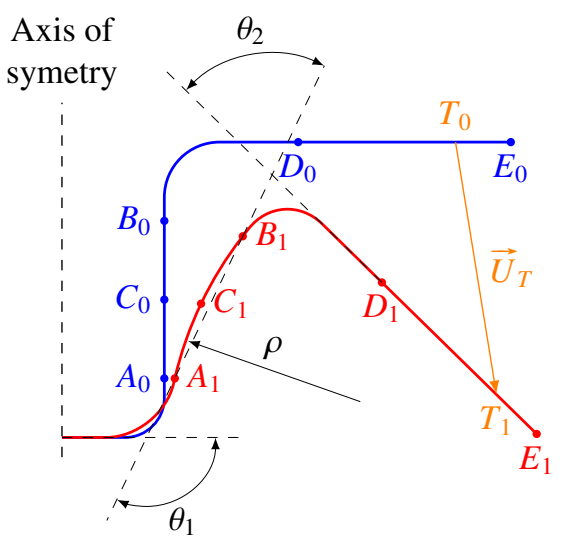

Figure 4. Parameters of the U-shape after spring-back

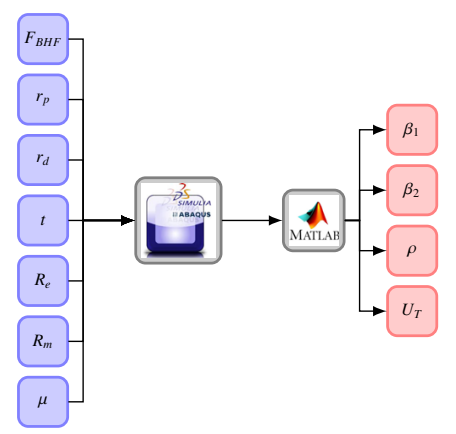

Figure 5. Please write your figure caption here

This numerical model is parametrized with the following 7 input parameters : the blank holder force $F_{B H F}$, the friction coefficient between tools and the blank $\mu$, the material properties of the DP780 steel Yield strength $R_{e}$, Tensile strength $R_{m}$, the thickness of the blank $t$ and the radii of die $r_{d}$ and of punch $r_{p}$.

The output parameters under consideration include the angles of $\theta_{1}, \theta_{2}$, the side wall curl $\rho$ and the displacement of a virtual hole $U_{T}$. These output parameters are computed with MATLAB ${ }^{\mathrm{TM}}$ (Fig. 5).

\subsection{Validation of the numerical model}

In the numisheet 2011 benchmark [8] experimental measurements are reported and compared with the set of results from several simulation results. Table 2 shows the results of our model for values of parameters in Table 1 . It can be observed, that there is rather small differences between our numerical results and experimental measurements from the numisheet 2011 benchmark.

It should be noticed that our model gives good results for the configuration of parameters given in Table 1 . It is of course too expensive, to obtain experimental measurements for a wide range of paramter's values. So we must thrust in the numerical model to explore the physical response and specifically in terms of spring-back. In the robust optimization process developed further, it is assumed that the numerical model gives consistent response for spring-back effects.

Table 1. Input data for comparison with experimental results of Numisheet 2011.

\begin{tabular}{lr}
\hline Input parameters & \\
\hline Blank thickness & $1.4 \mathrm{~mm}$ \\
Yield strength & $527 \mathrm{MPa}$ \\
Tensile strength & $831.5 \mathrm{MPa}$ \\
Friction coef. & 0.1 \\
Blank Holder force & $2940 \mathrm{~N}$ \\
Die radius & $7 \mathrm{~mm}$ \\
Punch radius & $5 \mathrm{~mm}$ \\
\hline
\end{tabular}

Table 2. Our numerical results and experimental measurements from Numisheet 2011.

\begin{tabular}{ccc}
\hline $\begin{array}{c}\text { Spring-back } \\
\text { parameters }\end{array}$ & $\begin{array}{c}\text { Numerical } \\
\text { results }\end{array}$ & $\begin{array}{c}\text { Experimental } \\
\text { measurements [8] }\end{array}$ \\
\hline$\theta_{1}$ & $108.32^{\circ}$ & $110.62^{\circ}$ to $118.3425^{\circ}$ \\
$\theta_{2}$ & $75.228^{\circ}$ & $77.61^{\circ}$ to $80.00^{\circ}$ \\
$\rho$ & $137.02 \mathrm{~mm}$ & $118.36 \mathrm{~mm}$ to $120.00 \mathrm{~mm}$ \\
\hline
\end{tabular}

\section{Robust optimization problem and meta-modelling}

For this case study, we are interested in the effect of springback on the displacement of a virtual hole $U_{T}$ on this U-shape part (Fig. 4). The goal is to evaluate the consequence of input parameters uncertainties on the hole displacement. All input parameters will be modelled as aleatory variables with normal distribution. Table 3 shows details of normal distributions used to model aleatory variables.

This problem will be formulated as a robust optimization problem, where the deviation of expected value of the displacement from a target is minimized, and where the standard deviation of the hole displacement will be also minimized. Among the 7 input parameters, $F_{B H F}, r_{d}$ and $r_{p}$ will be chosen as design variables $X$ (i.e controllable parameters), and the other 4 parameter $\left(\mu, R_{e}, R_{m}, t\right)$ will be 
Table 3. Probabilistic modelling and representation of uncertainties in the input parameters

\begin{tabular}{lllll}
\hline Param. & Unit & Dist. & $\begin{array}{l}\text { Std. dev. } \\
\sigma\end{array}$ & $\begin{array}{l}\text { Admissible } \\
\text { Mean val. }\end{array}$ \\
\hline$F_{B H F}$ & $\mathrm{kN}$ & Normal & 0.6667 & $2.94-50$ \\
$r_{d}$ & $\mathrm{~mm}$ & Normal & 0.0167 & $2-10$ \\
$r_{p}$ & $\mathrm{~mm}$ & Normal & 0.0167 & $2-10$ \\
$R_{e}$ & $\mathrm{MPa}$ & Normal & 16.667 & $500-600$ \\
$R_{m}$ & $\mathrm{MPa}$ & Normal & 20 & $780-900$ \\
$\mu$ & & Normal & 0.0033 & $0.04-0.16$ \\
$t$ & $\mathrm{~mm}$ & Normal & 0.0167 & $1-2$ \\
\hline
\end{tabular}

considered as noise parameters $P$ (i.e. uncontrollable parameters). This robust optimization problem can be stated as:

$$
\left\{\begin{array} { l } 
{ \operatorname { m i n } _ { X } | E [ U _ { T } ( X , P ) ] - U _ { T } ^ { \text { Cible } } | } \\
{ \text { and } } \\
{ \operatorname { m i n } _ { X } \sigma [ U _ { T } ( X , P ) ] }
\end{array} \text { with : } \left\{\begin{array}{l}
X=\left\{F_{B H F}, r_{d}, r_{p}\right\} \\
P=\left\{R_{e}, R_{m}, t, \mu\right\}
\end{array}\right.\right.
$$

The evaluation of the displacement of the virtual hole $U_{T}$ for a $(X, P)$ is based on the numerical model as presented previously and requires $2.5 \mathrm{~h}$ of calculation on a workstation running at $2.4 \mathrm{GHz}$.

In order to solve this optimization problem it is necessary to build a meta-model and set up an Design Of Experiment. We have defined a DOE based on a full factorial DOE with 3 levels for 7 parameters along a DOE of 2 supplementary levels for 2 parameters $\left(F_{B H F}, \mu\right)$. The overall DOE represent $3^{7}+\left(3^{5} \times 2^{2}\right)=3159$ numerical simulations (see Table 4). The huge amount of simulations has been ran on a parallel computer with 1600 CPU's.

Table 4. Factors and levels for the DOE

\begin{tabular}{ll}
\hline Param. & levels \\
\hline$F_{B H F}$ & $2.94,14.705,26.47,38.325,50 \mathrm{kN}$ \\
$r_{d}$ & $2,6,10 \mathrm{~mm}$ \\
$r_{p}$ & $2,6,10 \mathrm{~mm}$ \\
$R_{e}$ & $500,550,600 \mathrm{MPa}$ \\
$R_{m}$ & $780,840,900 \mathrm{MPa}$ \\
$\mu$ & $0.04,0.07,0.1,0.13,0.16$ \\
$t$ & $1,1.5,2 \mathrm{~mm}$ \\
\hline
\end{tabular}

Table 5. Comparison of accuracy of meta-models for $U_{T}$

\begin{tabular}{lll}
\hline Meta-model & MAE & $R^{2}$ \\
\hline Kriging & $1.0258 \times 10^{-4}$ & 0.9999 \\
SVD2 & 36.6112 & 0.8268 \\
SVD3 & 38.6366 & 0.9370 \\
RBF & $5.7494 \times 10^{-10}$ & 1 \\
NN & 16.9244 & 0.9988 \\
\hline
\end{tabular}

Here several meta-models have been tested. The two metrics which are used to measure the meta-models' accuracy are the R-square $\left(R^{2}\right)$ and the MAE.

$$
\mathrm{MAE}=\max \left|y_{i}-\hat{y}_{i}\right|, \quad i=1 \ldots m
$$

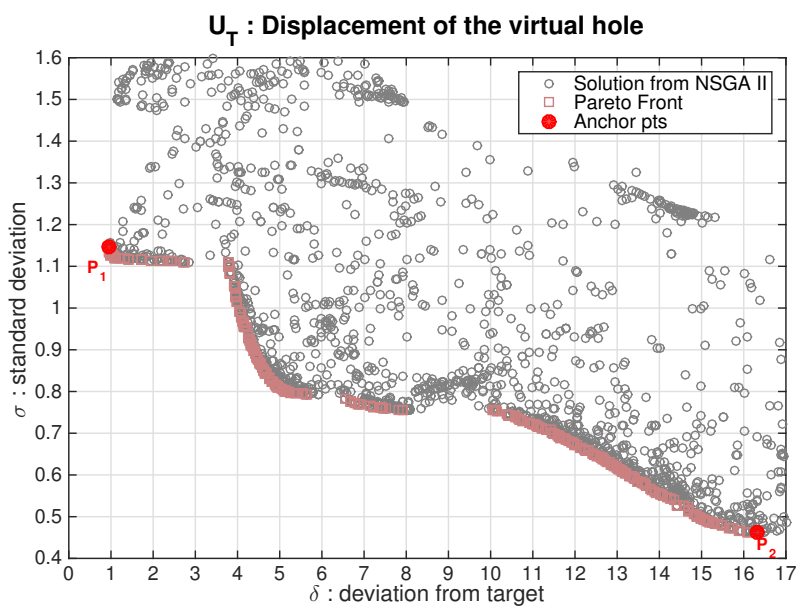

Figure 6. Please write your figure caption here

$$
R^{2}=1-\frac{\sum_{i=1}^{m}\left(y_{i}-\hat{y}_{i}\right)^{2}}{\sum_{i=1}^{m}\left(y_{i}-\bar{y}\right)^{2}}
$$

where $m$ is the number of validation points; $\hat{y}_{i}$ is the predicted value for the observed value $y_{i} . \bar{y}$ is the mean of the observed values at the validation points. The accuracy of each kind of metamodels including Kriging, Singular Value Decomposition of degree 2 (SVD2), Singular Value Decomposition of degree 3 (SVD3), Radial Basis Functions (RBF) and Neural Networks (NN) is given in Table 5. It is clearly found that the accuracy of RBF is the best both in terms of R-square and in terms of MAE in this case study. Therefore, the meta-models built by using the RBF will be used.

\section{Optimization Results}

The optimization process uses the stochastic optimization algorithm NSGAII [9], in the ModeFrontier ${ }^{\mathrm{TM}}$ software. The uncertainty propagation used here, is based on Latin Hypercube sampling with 1000 samples. Table 6 presents mean values and standard deviation of noise parameters used in the optimization problem modelling. The initial population comprises 125 individuals, and we ran 20 generations for the NSGAII algorithm.

The figure 6 shows the Pareto front obtained with the NSGAII algorithm. This Pareto front has 425 solutions. It can be seen that the two optimization criteria (deviation of expected value from target and standard deviation of hole displacement) are antagonist, thus this optimization problem is an actual robust optimization problem [10].

This optimization result has been obtained with the following noise parameters from Table 6 .

Tables 7 and 8 give the solution of the two "anchor" points of the Pareto front, i.e. the minimum mean and the maximum mean values of $U_{T}$. For each of these optimal solutions, the value of design variables $X$ is given. We can see that for the maximum mean value of $U_{T}$ (Table 8) we have the minimum standard deviation for $U_{T}$, so under the uncertainty of noise parameters this optimum value is very stable. On the other hand, for the minimum value of 
$U_{T}$ (Table 7) we have the maximum standard deviation for $U_{T}$, so this optimum solution is not stable.

These results demonstrate that one can control the effect of uncertainty on input parameter. By controlling the blank holder force $F_{B H F}$ we can reduce the effects of uncertainty of the hole displacement $U_{T}$.

Table 6. Mean and standard deviation values of noise parameters

\begin{tabular}{lll}
\hline Param. & Mean & Std. dev. \\
\hline$R_{e}[\mathrm{MPa}]$ & 550 & 16.667 \\
$R_{m}[\mathrm{MPa}]$ & 840 & 20 \\
$\mu[]$ & 0.1 & 0.0033 \\
$t[\mathrm{~mm}]$ & 2 & 0.0167 \\
\hline
\end{tabular}

Table 7. Optimization result for anchor point $P_{1}$

\begin{tabular}{lllll}
\hline Param. & Mean & Std. dev. & Min & Max \\
\hline$F_{B H F}[\mathrm{kN}]$ & 47.06 & 0.66 & 44.99 & 49.18 \\
$r_{d}[\mathrm{~mm}]$ & 9.950 & 0.017 & 9.898 & 10.003 \\
$r_{p}[\mathrm{~mm}]$ & 2.809 & 0.017 & 2.746 & 2.862 \\
$U_{T}[\mathrm{~mm}]$ & 0.9541 & 1.1475 & -2.3650 & 4.6207 \\
\hline
\end{tabular}

Table 8. Optimization result for anchor point $P_{2}$

\begin{tabular}{lllll}
\hline Param. & Mean & Std. dev. & Min & Max \\
\hline$F_{B H F}[\mathrm{kN}]$ & 19.23 & 0.66 & 17.16 & 21.36 \\
$r_{d}[\mathrm{~mm}]$ & 4.042 & 0.017 & 3.990 & 4.095 \\
$r_{p}[\mathrm{~mm}]$ & 4.914 & 0.017 & 4.851 & 4.966 \\
$U_{T}[\mathrm{~mm}]$ & 16.3225 & 0.4614 & 14.4989 & 17.9437 \\
\hline
\end{tabular}

\section{Conclusions}

In this paper we have proposed a robust optimization procedure of a draw bending process. In this work, uncertainties on the material properties, geometry of the blank and process's parameters have been modelled. Then among the set of parameters we have chosen design variables and noise parameters. This choice depend on the optimization context, here we have supposed that the geometry of the blank the material have been set previously in the design process of the part. Finally, assuming that the numerical model is consistent, the optimization results show that we can reduce effect of uncertainties by controlling the blank holder force.

\section{References}

1. M. Strano, Proceedings of the Institution of Mechanical Engineers, Part B: Journal of Engineering Manufacture 220, 1305 (2006)

2. W. Zhang, R. Shivpuri, Reliability Engineering \& System Safety 94, 152 (2009)

3. G. Gantar, K. Kuzman, Journal of materials processing technology 167, 237 (2005)

4. T. Buranathiti, J. Cao, Z.C. Xia, W. Chen, Probabilistic design in a sheet metal stamping process under failure analysis, in NUMISHEET 2005: Proceedings of the 6 th International Conference and Workshop on Numerical Simulation of 3 D Sheet Metal Forming Processes; Part A (2005), Vol. 778, pp. 867-872

5. G.J. Park, T.H. Lee, K.H. Lee, K.H. Hwang, AIAA journal 44, 181 (2006)

6. C. Zang, M. Friswell, J. Mottershead, Computers \& structures 83, 315 (2005)

7. J.H. Wiebenga, Ph.D. thesis (2014)

8. H. H, C. K, H. SS, C. WJ, Proceedings of Numisheet 2011 (2011)

9. K. Deb, S. Agrawal, A. Pratap, T. Meyarivan, Lecture notes in computer science 1917, 849 (2000)

10. V.D. Nguyen, Ph.D. thesis, Université de technologie de Troyes, ICD-LASMIS UMR 6281 (2015) 